\title{
Gyrokinetic Simulations of Short-Wavelength ITG Instability in the Presence of a Static Magnetic Island ${ }^{*)}$
}

\author{
Paul P. HILSCHER, Kenji IMADERA, Jiquan LI and Yasuaki KISHIMOTO \\ Graduate School of Energy Science, Kyoto University, Gokasho, Uji, Kyoto 611-0011, Japan
}

(Received 23 November 2012 / Accepted 18 February 2013)

\begin{abstract}
The ion-temperature-gradient (ITG) mode instability in sheared slab geometry in the presence of a mesoscale magnetic island is investigated with a focus on the short-wavelength regime $\left(k_{y} \rho_{i}>1\right)$. We found that a static magnetic island causes radial and poloidal modes to couple and therefore plays a stabilizing role by allowing energy transfer from unstable modes to stable modes. However, for large island widths, the short-wavelength ITG mode becomes strongly destabilized and tends to dominate over the standard ITG mode $\left(k_{y} \rho_{i} \simeq 0.5\right)$. A reduced model is proposed in this paper to understand this destabilization mechanism.
\end{abstract}

(c) 2013 The Japan Society of Plasma Science and Nuclear Fusion Research

Keywords: gyrokinetics, ITG instability, magnetic island, numerical simulation, microturbulence

DOI: $10.1585 /$ pfr. 8.2403040

\section{Introduction}

The short-wavelength ion-temperature-gradient (swITG) mode was discovered by Smolyakov et al. [1] in shearless slab geometry as a second destabilization of the ITG mode for large poloidal mode numbers $k_{y} \rho_{i}>1$. Follow up investigation by Gao et al. confirmed that the swITG mode exists in sheared slab geometry [2], as well as in toroidal geometry [3]. Gao et al. also found that a large electron temperature gradient $\eta_{e}$ has a destabilizing effect on the sw-ITG mode, and that while a large magnetic shear $\hat{s}$ and plasma pressure $\beta$ have a stabilizing effect, it still cannot completely suppress the sw-ITG mode. Recently, it was found that although the linear growth rate of the sw-ITG mode is comparable to the usual long-wavelength standard ITG (std-ITG) mode, its contribution to the heat flux in the non-linear regime is negligible [4].

In this study, we are investigating the effect from the equilibrium magnetic field perturbation arising from a static magnetic island on an ITG drift wave in the linear phase. This setup was also studied by Wang et al. [5], who found that islands of size $w<10 \rho_{i}$ have a stabilizing role arising from an induced poloidal mode coupling, which enables the dissipation of energy by stable modes. On the other hand, for larger magnetic islands widths $\left(w>10 \rho_{i}\right)$, the destabilizing effect due to the appearance of additional rational surfaces dominates. However, it should be noted that these results were obtained using a gyrofluid simulation model that overestimates the damping and does not take the sw-ITG mode into account. It was therefore necessary to reconfirm these findings using a more sophisticated simulation model.

By performing gyrokinetic simulations, we found

author'se-mail: paul@center.iae.kyoto-u.ac.jp

*) This article is based on the presentation at the 22nd International Toki Conference (ITC22). that, although poloidal coupling can transfer energy to stable modes which is then dissipated away through Landau damping and finite Larmor radius (FLR) effects, the stabilization effect is reduced owing to the appearance of the sw-ITG mode. In addition, for large magnetic island widths, the sw-ITG mode is also more sensitive to the modification of the equilibrium magnetic geometry, and achieves a growth rate exceeding that of the std-ITG mode. The global mode which is formed because of the poloidal mode coupling is then dominated by the sw-ITG mode.

\section{Basic Equations}

We employ the gyrokinetic equations in a twodimensional sheared slab geometry using the local approximation, where a constant temperature throughout the domain is assumed but with a finite temperature length scale $L_{T}$ and a density gradient $L_{n}$. The distribution function is split into a constant Maxwellian part $f_{0}=\exp \left(-v_{\|}^{2}-\mu\right)$ and the perturbed part $f_{1}\left(x, k_{y} ; v_{\|}, \mu\right)$. Here, $x$ is the radial direction, whereas the poloidal direction $y$ is decomposed into Fourier harmonics $k_{y}$. The variables $v_{\|}$and $\mu$ are the parallel velocity and magnetic moment, respectively. The linearized Vlasov equation is written as

$$
\begin{aligned}
\frac{\partial f_{1}}{\partial t}= & i k_{y}\left\{1+\eta_{i}\left(v_{\|}^{2}+\mu-\frac{3}{2}\right)\right\}\langle\phi\rangle f_{0} \\
& +i k_{\|} v_{t h} v_{\|}\left(f_{1}+\langle\phi\rangle f_{0}\right) \\
& -v_{t h} v_{\|}\left[\psi_{1}, f_{1}+\langle\phi\rangle f_{0}\right]+C_{L B},
\end{aligned}
$$

with $\eta_{i}=L_{T} / L_{n}$ and the thermal velocity defined as $v_{t h}=\sqrt{2 T_{i} / m_{i}}$. Also, $\langle A\rangle$ denotes the gyroaveraging and is defined as $\langle A\rangle=J_{0}(\lambda) A$ with $\lambda=\sqrt{2 \rho_{t h} \mu k_{\perp}^{2}}$, where $J_{0}$ is the Bessel function of the first kind. The physical quantities are normalized as $x \rho_{i} \rightarrow x, k_{y} / \rho_{i} \rightarrow$ $k_{y}, v_{\|} / v_{t h}=v_{\|}, f_{0} v_{t h} / n_{0} \rightarrow f_{0}, f_{1} L_{n} v_{t h} / \rho_{t h} n_{0} \rightarrow f_{1}$, 


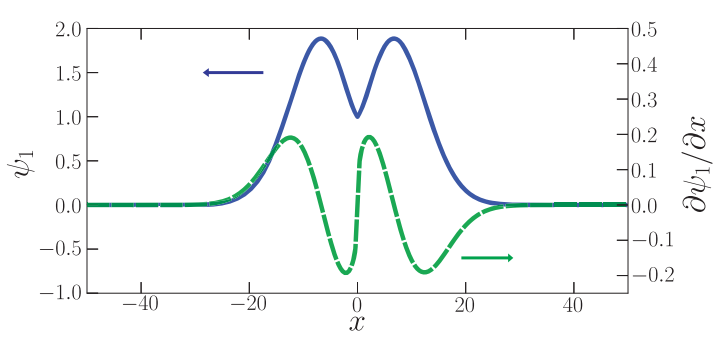

Fig. 1 The magnetic flux eigenfunction $\psi_{1}$ and its derivative $\partial \psi_{1} / \partial x$ of the tearing mode instability with $m=1 \mathrm{ob}-$ tained from a numerical simulation using a Harris sheet profile.

$t v_{t h} / L_{n} \rightarrow t, \phi e L_{n} / v_{t h} \rightarrow \phi, \mu B / m v_{t h}^{2} \rightarrow \mu, \rho_{t h}=v_{t h} / \Omega_{i}$ and $\Omega_{i}=-e B / m_{i} c$. In standard sheared slab geometry without a magnetic island, the parallel wavenumber is expressed as $k_{\|}=\hat{s} x k_{y}$, where $x=0$ is equivalent to the position of the rational surface. The third term in Eq.(1) is the geometrical effect which arises from a static magnetic perturbation $\psi_{1}$, where $[A, B]$ is the Poisson bracket, defined as $\partial_{x} A \partial_{y} B-\partial_{y} A \partial_{x} B$. A Lenard-Bernstein collisional model $C_{L B}$, with a small collisionality $\beta_{C}=10^{-3}$ is included,

$$
C_{L B}=\beta_{C}\left(f_{1}+v_{\|} \partial_{v_{\|}} f_{1}+\partial_{v_{\|}}^{2} f_{1}\right),
$$

in order to reproduce the Landau damping of stable modes. The Poisson equation is

$$
\begin{gathered}
\left\{\lambda_{D}^{2} k_{\perp}^{2}+\frac{1}{T_{i}}\left(1-\Gamma_{0}\left(b_{i}\right)\right)+\frac{1}{T_{e}}\right\} \phi \\
=\int_{v_{\|}=-\infty}^{\infty} \int_{\mu=0}^{\infty}\left\langle f_{1}\right\rangle \mathrm{d} \mu \mathrm{d} v_{\|},
\end{gathered}
$$

with $\Gamma_{0}(b)=I_{0}(b) e^{-b}$, and $I_{0}$ the modified Bessel function of the first kind, zeroth order, $b=\rho_{t h}^{2} k_{\perp}^{2}$ and $\lambda_{D}$ is the Debye length. Debye length effects are neglected as $\lambda_{D}^{2} / \rho_{i}^{2} \ll 1$. The electrons are assumed to be adiabatic with a temperature of $T_{e}=T_{i}$.

For the magnetic perturbation $\psi_{1}$ the magnetic flux eigenfunction of a single tearing mode is used, which is calculated using a reduced MHD model, (see e.g. Strauss [6]). A Harris current sheet $\psi_{\mathrm{eq}}=\tanh (\hat{s} x)$ is chosen as the initial equilibrium for the MHD simulation. For this setup, a tearing mode instability with poloidal mode number $m=1$ is unstable with a magnetic flux eigenfunction $\psi_{1}$, as shown in Fig. 1. We note that the evolution of the magnetic island can be considered slow compared to the gyrokinetic drift wave timescale and thus the assumptions for keeping the temperature and density gradient are justified. Assuming that $\psi_{1}$ contributes only through the $m=1$ component of the magnetic island structure, $\psi_{1}$ is given by

$$
\psi_{1}=\tilde{\psi}(x) \frac{1}{2}\left\{\mathrm{e}^{i 2 \pi y / L_{y}}-\mathrm{e}^{-i 2 \pi y / L_{y}}\right\} .
$$

The Poisson bracket is directly calculated using the triad mode coupling condition

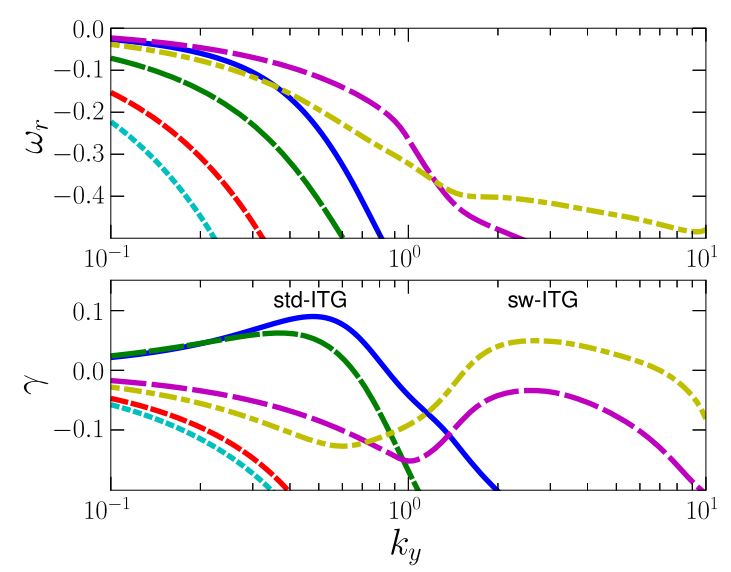

Fig. 2 Dispersion relation for $\hat{s}=0.2$ and $\eta_{i}=5$ and adiabatic electrons. Each line corresponds to a branch of the ITG mode. Two unstable std-ITG branches exist for $k_{y}<1$, as well as one unstable sw-ITG branch for $k_{y}>1.5$.

$$
\begin{aligned}
& {\left[\psi_{1}, f_{1}+\langle\phi\rangle f_{0}\right]=} \\
& \tilde{\psi}_{x}\left(k_{y}^{m+1} f_{1}^{m+1}+k_{y}^{m-1} f_{1}^{m-1}\right) \\
& +\tilde{\psi}_{x}\left(k_{y}^{m+1} \phi^{m+1}+k_{y}^{m-1} \phi^{m-1}\right) f_{0} \\
& +\tilde{\psi} k_{y}^{1}\left(f_{1, x}^{m+1}-f_{1, x}^{m-1}\right) \\
& +\tilde{\psi} k_{y}^{1}\left(\phi_{x}^{m+1}-\phi_{x}^{m-1}\right) f_{0}
\end{aligned}
$$

where $\tilde{\psi}_{x}=\partial_{x} \tilde{\psi}$ and $k_{y}^{m}=2 \pi m / L_{y}$. The Poisson bracket term also includes contributions from the neighboring poloidal modes, and thus poloidal modes are coupled once $w>0$. For the negative $k_{y}$ modes, the corresponding complex conjugate value of the positive mode is used.

\section{The Short-Wavelength ITG Mode}

The dispersion relation of the ITG instability with $\psi_{1}=0$ is calculated as a non-linear integral eigenvalue equation. A detailed overview of the solution procedure is given by Idomura [7]. Thus we will only present the results here. We use $\hat{s}=0.2, \eta_{i}=5$ to calculate the dispersion relation as shown in Fig. 2. We find multiple eigenmodes in the system with most of them being stable. For the std-ITG region with $k_{y}<1$, we can identify two unstable modes with similar growth rates, whereas for $k_{y}>1$, both these modes are stable. However, for $k_{y}>1.5$, a further ITG mode becomes unstable with a narrow radial eigenstructure. We refer to this mode as the sw-ITG mode. The peak growth rate of the sw-ITG mode is reached around $k_{y}=2.5$ with approximately half the growth rate of the std-ITG mode. We note that the sw-ITG mode also exists for kinetic electrons, although a finite electron temperature gradient is required in order to destabilize the mode.

\section{ITG Instability with a Static Mag- netic Island Structure}

We solved the gyrokinetic equation system Eq. (1) as 


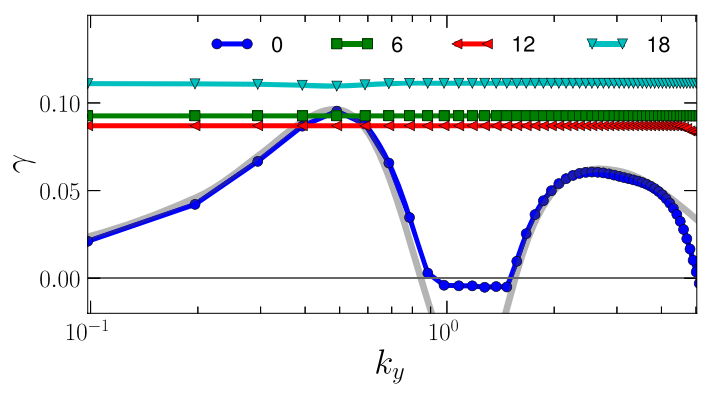

Fig. 3 Growth rates of poloidal modes for $w=0,6,12,18$. For $w>0$ all modes have an equal growth rates owing to the formation of a global mode. The gray line shows the theoretical growth rate for $w=0$.

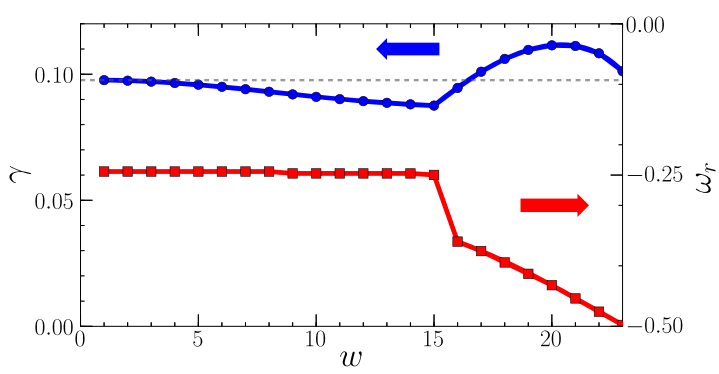

Fig. 4 Growth rate $\gamma$ and real frequency $\omega_{r}$ of the global mode depending on the magnetic island width $w$ for $\hat{s}=0.2$ and $\eta_{i}=5$. Strong destabilization is observed for $w>15$.

an initial value problem using the $g k c++$ code [8] and the parameters $\hat{s}=0.2$ and $\eta_{i}=5$. Once a finite magnetic island width is included ( $w>0$ ), a global mode is established through poloidal mode coupling, where all poloidal modes have equivalent growth rates (and real frequencies), as shown in Fig. 3. Coupling enables unstable modes to transfer energy to stable modes, where it is then dissipated away through finite Larmor radius effects and/or Landau damping [5]. This is observed as the reduction of the growth rate of the global mode. As a larger island size enhances the coupling between the modes, this stabilization mechanism increases for larger island sizes until a width of $w \approx 15$, as shown in Fig. 4. However, the stabilization mechanism is weak compared to that reported by Wang et al. [5]. This can be attributed to the appearance of the sw-ITG mode, which reduces the energy dissipation by providing a free-energy source, as can be seen by the contribution to the energy spectra in Fig. 6. Here, for small magnetic islands $w<15$, the ITG global mode structure is broadened, and mainly determined by the most unstable mode, see Figs. 5 (a)-(c). For large island widths $(w>15)$, a strong destabilization of the global mode is observed, together with a change in the global mode structure, which changes to a very localized and narrow structure as is shown in Fig. 5 (d). The origin of this destabilization can be attributed to the sw-ITG mode, which then drives the global mode as can be seen in the energy spectra

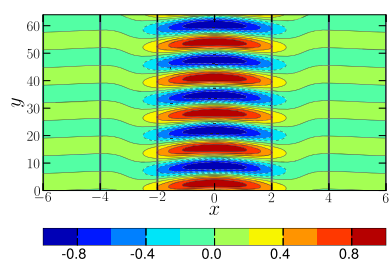

(a) $w=0$

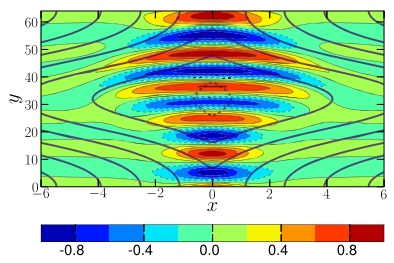

(c) $w=12$

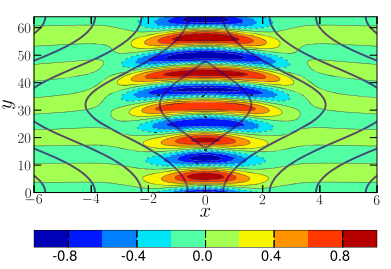

(b) $w=6$

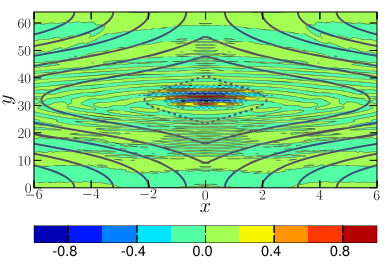

(d) $w=16$
Fig. 5 Contours of electric potential $\phi$ of the ITG global structure for island widths of $w=0,6,12,16$.

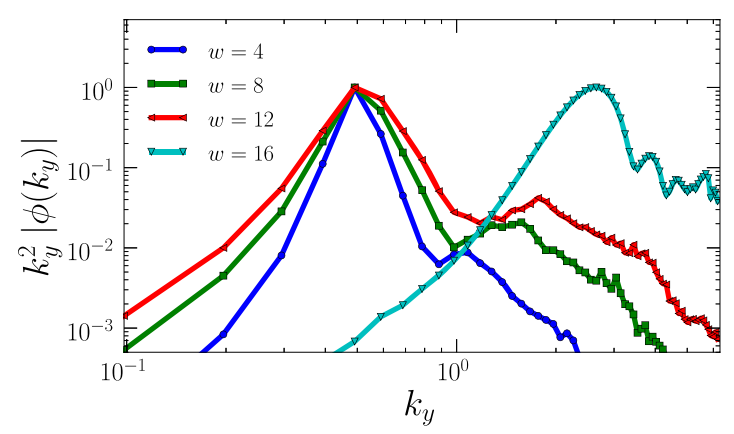

Fig. 6 Normalized energy spectra for island sizes of $w=$ $4,8,12,16$.

in Fig. 6. In addition, by artificially damping the sw-ITG mode in the simulations, we could confirm that the std-ITG mode is also destabilized, however for much larger magnetic island widths (i.e. $w \approx 20$ ). The physical mechanism of this destabilization is elucidated using a reduced model in the next section.

\section{Reduced Model}

In order to understand the destabilization of the swITG mode observed in Fig. 4 and Fig. 6, a reduced model is proposed in order to help analyze the response of the ITG mode to the static magnetic island. For simplicity, we fix the poloidal direction at $y=0$ and $y=L_{y} / 2$, which corresponds to the X-point and O-point of the island, respectively. This excludes the poloidal mode coupling from the island which arises from the islands poloidal structure in Eq.(4). The islands magnetic flux $\psi$ is now related only to the Landau damping and parallel advection term, as $k_{\|}=k_{y}\left(\hat{s} x \pm \tilde{\psi}_{x}\right) \equiv k_{y} B_{y}$. The modified magnetic field $B_{1 y}$ with contributions from the magnetic island and the equilibrium at the $\mathrm{X}$ - and O-point is shown in Fig. 7. At the $\mathrm{X}$-point, the island structure increases the local shear close to the rational surface. However, at the O-point, the 


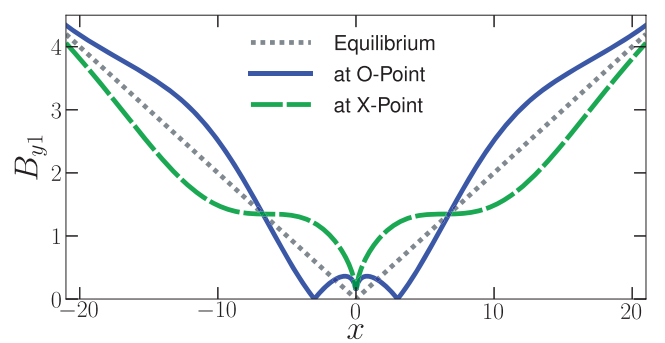

Fig. 7 Perturbation of the equilibrium field due to the magnetic island of size $w=20$ at the X-and O-point.

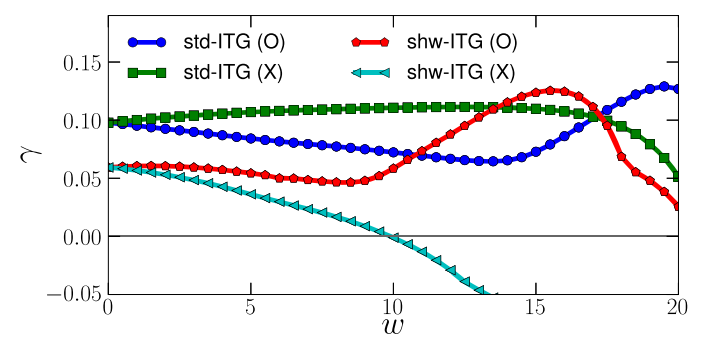

Fig. 8 ITG growth rates at the island's X-and O- point for $k_{y}=$ 0.5 and $k_{y}=2.5$ modes versus the island width $w$.

island leads to a separation of the rational surfaces, the distance of which increases with the size of the island. For the perturbed magnetic field at the $\mathrm{X}$ - and O-point, we evolve only a single poloidal mode, with a wavenumber which corresponds to the peak growth rate of the std-ITG mode at $k_{y} \approx 0.5$ and of the sw-ITG mode at $k_{y} \approx 2.5$. The growth rate's dependence on the island width is shown in Fig. 8. For the X-point equilibrium, the std-ITG mode is slightly destabilized, which agrees well with the general behavior of destabilization for weak magnetic shear and the stabilization for stronger shearing rates. The sw-ITG mode however is very sensitive to the increase in magnetic shear and quickly stabilizes. For the O-point equilibrium, the std-ITG mode is stabilized for small island widths on account of the reduction of local shear, however it is destabilized again for large island widths because of the separation of rational surfaces. As the island width increases and crosses a critical value, the std-ITG mode and also the sw-ITG mode are first destabilized, and then stabilized again, as shown in Fig. 8. However, the critical island width for the sw-ITG mode is smaller than that of the std-ITG. We can understand this underlying mechanism through the response of the ITG eigenmode structures to the island size. As shown in Fig. 7, for large islands, the rational surface is separated into two, so that a so-called double ITG mode with an increased growth rate is excited [9]. The corresponding eigenfunctions of the std-ITG and sw-ITG modes are shown in Fig. 9. As the island width increases further, the double ITG modes are destabilized first with the increase in the rational surface separation. Finally, when the separation is large enough, the double ITG mode structure also separates, so that the

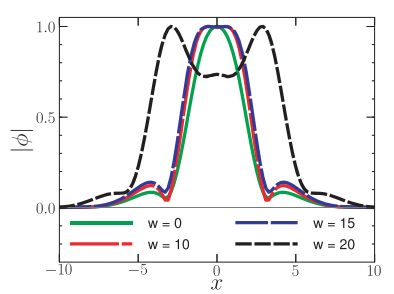

(a) std-ITG

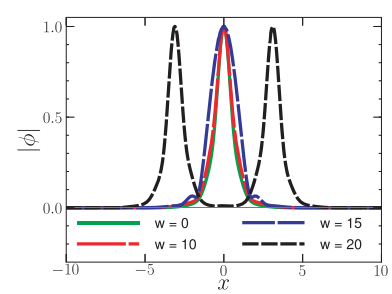

(b) sw-ITG
Fig. 9 Eigenfunctions (absolute value) of the std-ITG mode for $k_{y}=0.5$ and sw-ITG mode for $k_{y}=2.5$ for different island width.

mode structures reduce back to the eigenstructure with a single rational surface, as shown in Fig. 9, which is why the growth rate decreases. As for the difference of the critical island widths for the destabilization of the std-ITG and sw-ITG modes, this phenomenon may result from the narrow mode width of the sw-ITG mode, which matches the small separation of the rational surface. Hence, the critical island widths for the destabilization and even stabilization of the sw-ITG mode are reasonably smaller than those for the std-ITG mode as shown in Fig. 8.

\section{Conclusion}

We have studied the effect of equilibrium field perturbation arising from a static magnetic island. We have found that a small magnetic island has a stabilizing effect on the ITG mode, because it enables unstable poloidal modes to couple to stable modes and dissipate energy. However, for large islands the separation of the rational surfaces caused by the island destabilizes the ITG mode. In this case, the destabilization of the sw-ITG mode is dominant on account of its smaller radial mode structure, and is therefore more sensitive to rational surface separation compared to the stdITG mode.

\section{Acknowledgments}

This work was supported by the Grant-in-Aid from JSPS (No. 21340171 and No. 23840025).

[1] A.I. Smolyakov, M. Yagi and Y. Kishimoto, Phys. Rev. Lett. 89, 125005 (2002).

[2] Z. Gao, H. Sanuki, K. Itoh and J.Q. Dong, Phys. Plasmas 10, 2831 (2003).

[3] Z. Gao, H. Sanuki, K. Itoh and J.Q. Dong, Phys. Plasmas 12, 022502 (2005).

[4] J. Chowdhury, S. Brunner, R. Ganesh, X. Lapillonne, L. Villard and F. Jenko, Phys. Plasmas 19, 102508 (2012).

[5] Z.X. Wang, J.Q. Li, Y. Kishimoto and J.Q. Dong, Phys. Plasma 16, 060703 (2009).

[6] H.R. Strauss, J. Plasma Phys. 57, 83 (1997).

[7] Y. Idomura, S. Tokuda and M. Wakatani, Phys. Plasmas 6, 4658 (1999).

[8] P.P. Hilscher, K. Imadera, J.Q. Li and Y. Kishimoto, ZeroCarbon Energy Kyoto 2010 (Springer, 2011) p.239.

[9] J. Li, L. Huang and W. Qu, Phys. Plasmas 5, 959 (1998). 